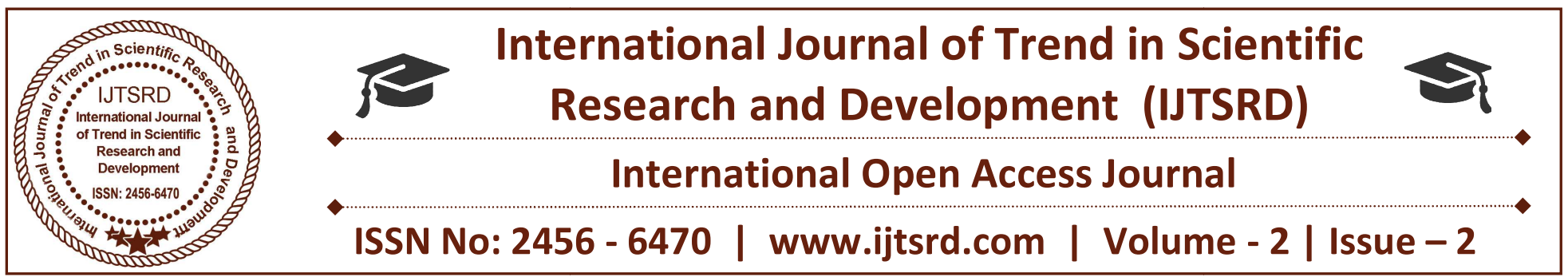

\title{
Automatic Car Window Opener for safe Driving
}

\author{
Dr. S Senthil Kumar, Anjali S, Hashina Parveen S, Aishwarya R \\ Electrical and Electronics Engineering, Bannari Amman Institute of Technology, \\ Sathyamangalam, Erode, Tamil Nadu, India
}

\section{ABSTRACT}

Gas leakage is the major problem in the automobile Sector. According to the researches, it is said that the car's dashboard, sofa, air freshner ,gas emitted in ac emits the harmful carcinogen, Benzene in cars. It damages our bones, reduces white blood cells and causes anemia. When these benzene mixes with the freon (refridgerent) used in air conditioner, it emits co gas. The level of this harmful gas is $50 \mathrm{mg}$ per square $\mathrm{ft}$. And it is found that the parked car with closed windows will contain $400-800 \mathrm{mg}$ per square $\mathrm{ft}$ of benzene. So we find it really harmful for us to inhale the excessive amount of toxin. However, most commercially available systems can detect only one gas. This paper is to introduce the new technique of opening the windows automatically to the half when the gas level exceeds its threshold level for exhausting those gases This paper aims at detection of the harmful gases using sensors, displaying the amount of gases present inside the car using the LCD display, alerting the inmates of the car with recorder IC and further the windows of car is opened. The project working status is also verified through LCD display and opens the windows automatically.

\section{INTRODUCTION}

The sensors play a crucial role since the detection of harmful gases is the foremost process. Sensors are the transducers which convert any quantity into electrical quantity. If the sensors are given the supply, they automatically detect the disturbances in the environment and react accordingly. The sensors are usually interfaced with the microcontrollers and are programmed according to the user's requirements, A hazardous gas detection has its applications suitable for ammonia, aromatic compounds, sulphur, benzene vapour, smoke and other gases harmful gas detection The voice IC is provided so that the voice could be recorded by us indicating the excessive amount of gases that has been beyond the threshold level. If the amount of gases inside the automobile is above the threshold value then the voice IC gives out the indication. In the other case the voice IC does not function .In this work, we mainly focussed on the indication of proper working of the project itself and also the opening of the car window automatically without the knowledge of the inmates and then with the voice message and with the buzzer. Here with the help of arduino we designed such a kit with the following characteristics and features to meet the requirements and demands of the individuals who are not aware of the harmful gases either because it is odourless or because the inmates are sleeping.

\section{BACKGROUND STUDY}

There are many published articles in the areas of the harmful gas detection. Some relevant papers are discussed in this section..If cars are parked outdoors under the sun at temperature above 60 degrees Farenheit the benzene level rises to 2000 to $4000 \mathrm{mg}$, 40 times the accepted level. The American chemical society released a report in 2007 that the automobile were found to contain "wide variety of synthetic materials that emits organic substances". Benzene is obtained from Coal and petroleum products majorly 
and most commonly. It is a colourless liquid with sweet smell that evaporates when it comes in contact with the air. Benzene is present in the smoke of cigarette, car exhausts, glues and paints according to the survey of Center for Disease Control (CDC).The $\mathrm{CDC}$ factsheet on benzene says that it can cause difficulty in thinking, change in heart function, unconsciousness and sometimes even death. This warning is been reported by Australia's Commonwealth Scientific and Industrial Research Organization (CSIRO).In December 19,2001 report, the organisation found "high levels of air toxic emissions in new motor vehicles for upto six months and longer after they leave the showroom". That research, however was conducted on three automobiles manufactured in Australia and one imported .Benzene was first reported to induce cancer in humans in the 1920's. The chemical industry claims that it wasn't until 1979 that it was determined "conclusively" in humans. Benzene has been shown to cause cancer, saying that they occur in both the sexes of multiple species of laboratory animals exposed through various ways.

\section{BLOCK DIAGRAM:}

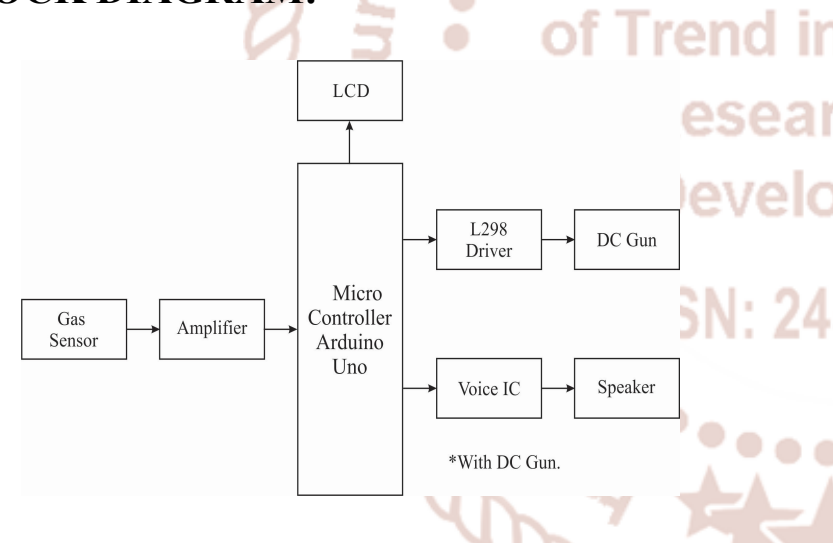

Figure 1.Block diagram

\section{BLOCK DIAGRAM DESCRIPTION}

\section{ARDUINO UNO:}

Arduino's processor basically uses the Harvard architecture where the program code and program data have separate memory. It consists of two memories- Program memory and the data memory. The code is stored in the flash program memory, whereas the data is stored in the data memory. The Atmega328 has $32 \mathrm{~KB}$ of flash memory for storing code (of which $0.5 \mathrm{~KB}$ is used for the boot loader), 2 $\mathrm{KB}$ of SRAM and $1 \mathrm{~KB}$ of EEPROM and operates with a clock speed of 16MHz.A typical example of
Arduino board is Arduino Uno. It consists of ATmega328- a 28 pin microcontroller.

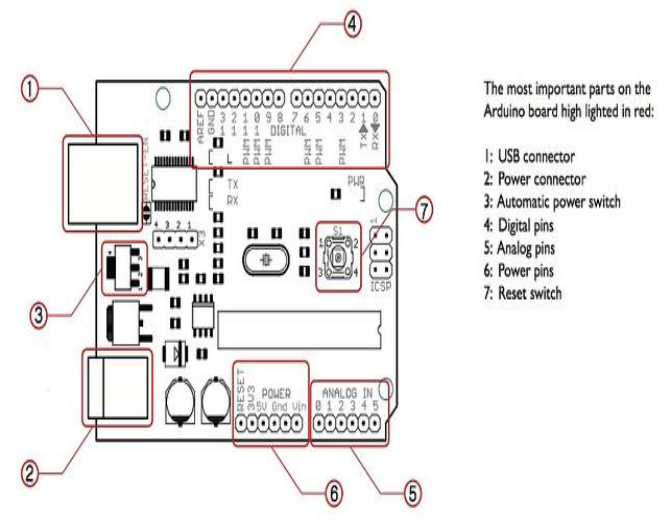

Figure2:Arduino pro mini

\section{GAS SENSOR MQ5:}

MQ-5 Semiconductor Sensor for Combustible Gas Sensitive material of MQ-5 gas sensor is $\mathrm{SnO} 2$, which with lower conductivity in clean air. When the target combustible gas exist, the sensors conductivity is higher along with the gas concentration rising. We use simple electro-circuit, convert change of conductivity to correspond output signal of gas concentration. MQ5 gas sensor has high sensitivity to Methane, Propane and Butane and could be used to detect both Methane and Propane. The sensor could be used to detect different combustible gas especially Methane, it is with low cost and suitable for different application.

\section{LCD SCREEN:}

A liquid crystal display (LCD) is convenient for electronic visual display or video display that uses the light modulating properties of liquid crystals (LCs). LCs does not emit light directly.LCD is used to display the parameters.

\section{SPEAKER:}

The speaker takes the power supply from relay. The arduino sends signals to the speaker as needed. The frequency can be assigned to the speaker so that sensor can be assigned with sound. The speaker is when the level of gas exceeds the threshold value.

\section{GUN:}

A dc gun is something that converts energy into motion. It also can be used to apply a force. An actuator typically is a mechanical device that takes energy usually energy that is created by air, electricity or liquid and converts it into some kind of motion. That motion can be in virtually any form, such as blocking, clamping or ejecting. Actuators typically are 
used in manufacturing or industrial applications and might be used in devices such as motors, pumps, switches and valves. Thus in this project it is used in the motion of opening car window automatically. In this project we use Linear actuator.

\section{SD 1820 SOUND/VOICEBOARD RECORDING RECORDER PLAYBACK MODULE:}

This module board is based on ISD18B20, which is a single-chip single-message record/playback device. Recordings are stored into on-chip non-volatile memory, providing zero-power message storage. With the embedded Flash memory employed, data retention up to 100 years and typical 100,000 erase/record cycles can be reached. Time for recording is 8-20 seconds.

\section{L298 DRIVE:}

The L298 is an integrated monolithic circuit in a 15lead Multi watt and PowerSO20 packages. It is a high voltage, high current dual full-bridge driver designed to accept standard TTL logic levels and drive inductive loads such as relays, solenoids, DC and stepping motors. Two enable inputs are provided to enable $r$ disable the device independently of the input signals. The emitters of the lower transistors of each bridge are connected together and the corresponding external terminal can be used for the connection of an external sensing resistor. An additional supply input is provided so that the logic works at a lower voltage.

\section{AMPLIFIER:}

An electronic amplifier is a device for increasing the power of a signal. It does this by taking energy from a power supply and controlling the output to match the input signal shape but with a larger amplitude. In this sense, an amplifier may be considered as modulating the output of the power supply.

\section{POWER SUPPLY:}

The power supply should be of $+5 \mathrm{~V}$, with maximum allowable transients of $10 \mathrm{mv}$. To achieve a better / suitable contrast for the display, the voltage (VL) at pin 3 should be adjusted properly. A module should not be inserted or removed from a live circuit. The ground terminal of the power supply must be isolated properly so that no voltage is induced in it. The module should be isolated from the other circuits, so that stray voltages are not induced, which could cause a flickering display.

\section{PROPOSED SYSTEM}

In the proposed automatic car window opener system, the detection system along with the automation is employed with the help of various components. The system alerts the inmates of the excess abnormal gases with the help of LCD displays and the buzzer is on in order to alert the persons exposed to it. In addition, voice control is provided to inform the present state of the person. If the value of the poisonous gas exceeds the reference value, it alerts and then opens the window automatically by itself. Thus the gases are exhausted.

The operations of the proposed system are that when the harmful gases detected in the sensor senses these gases, it sends the information to the arduino and then the values are continuously displayed on the LCD display. At this instant the actuator does not experience any changes in it and thus remains closed. But when the harmful gas level exceeds the threshold value the buzzer and the voice IC intimates the inmates and another LCD display indicating the proper working of the project is verified. So that proper measures are taken as precaution. The proposed system is implemented by using dot board. The code is written in C. The Arduino UNO used in this project is low cost, easy to program, flexible to add new functions, and can be reprogrammed with no additional costs. Arduino is a single-board microcontroller. The hardware features with an opensource hardware board designed around an 8-bit Atmel AVR microcontroller or a 32-bit Atmel ARM. Current models consists a USB interface, 6 analog input pins and 14 digital $\mathrm{I} / \mathrm{O}$ pins that allows the user to attach various extension boards. It is a $16 \mathrm{MHz}$ clock which is fast enough for most applications and does not speeds up the microcontroller. The MQ series of gas sensors use a small heater inside with an electro-chemical sensor. They are sensitive for a range of gasses and are used indoors at room temperature. The output is an analog signal and can be read with an analog input of the Arduino. The MQ-2 Gas Sensor module is useful for gas leakage detecting in home and industry. It can detect LPG, i-butane, propane, methane, alcohol, hydrogen and smoke. The sensor, thus detects and respond to some type of input from the physical environment. The specific input could be light,heat,motion ,moisture, pressure, or any of a great number of other environmental phenomena. The output is generally a signal that is converted to human readable display using the LCD screen. In the presence of gas which exceeds the threshold level, the 
arduino sends the signal to the buzzer and the level of gas is displayed. Here the voice recorder is also used to alert the inmates.

\section{OUTPUT OF SIMULATION}

The simulation results when the door is closed and the system operating under normal condition.

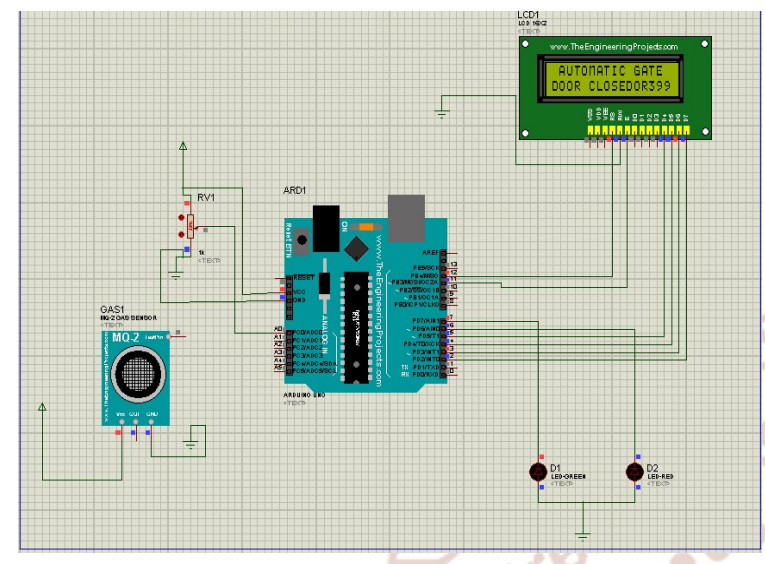

Figure 3 : simultion output I

The simulation results when the door is open and the system operating under gaseous condition

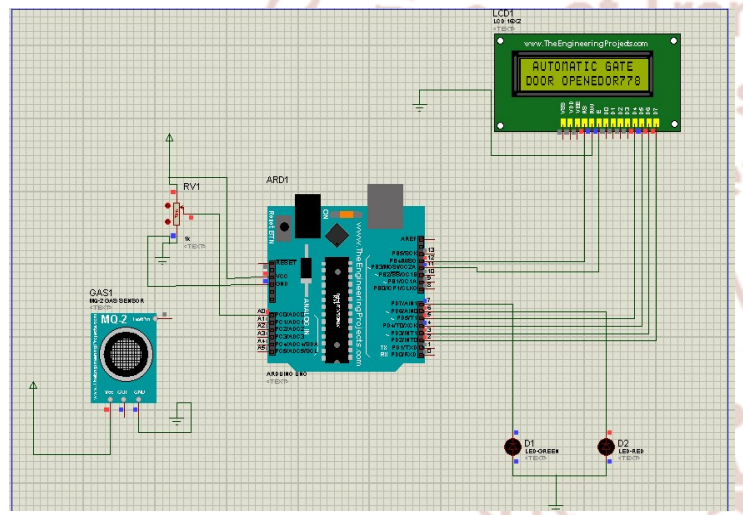

Figure 4: simulation output II

\section{CONCLUSION}

In the automobiles there may be emission of poisonous gases with or without knowledge. Maintainance of vehicles is one of the ways to avoid such accidents. But in all the cases it may not be possible. So this proposed system gives us assurance of being safe from the harmful poisonous gases. This system therefore detects the gases and indicates the inmates about the exceeding poisonous gas level. This system uses the sensors and actuators for sensing and automatically opening of the car window. Future extensions can also deal with the simplification of this module and embedding it into a small electronic system.

\section{REFERENCES}

1) M. Gao, F. Zhang, and J. Tian, "Environmental monitoring system with wireless mesh network based on embedded system", in Proc. $5^{\text {th }}$ IEEE Int. Symp. Embedded Computing, pp. 174-179, 2008.

2) L. Shaw, S. Bagha,A. G. Mahapatraand N. Nayak, "Kernel Approach on Detection of The Ethanol connection using Zno Gas Sensor ," International Journal of Machine Learning and computing, vol. 2, no. 1, Feb. 2012.

3) I. Morsi, "Electronic Noses for Monitoring Environmental Pollution and Building Regression Model," IEEE, 2008. 